\title{
Improved antiviral efficacy using TALEN-mediated homology directed recombination to introduce artificial primary miRNAs into DNA of hepatitis B virus
}

Timothy Dreyer ${ }^{1,2}$, Samantha Nicholson ${ }^{1,3}$, Abdullah Ely, Patrick Arbuthnot, Kristie Bloom ${ }^{*}$

SA-MRC/Wits Antiviral Gene Therapy Research Unit, Faculty of Health Sciences, University of the

Witwatersrand, Private Bag 3, Wits, 2050, Johannesburg, South Africa

\footnotetext{
${ }^{1}$ These authors are joint first authors on this work.

${ }^{2}$ Current affiliation: UP Biomedical Research Centre, Faculty of Veterinary Sciences, University of Pretoria, South Africa.

${ }^{3}$ Current affiliation: SA-MRC/UP Institute for Cellular and Molecular Medicine, Faculty of Health Sciences, University of Pretoria, South Africa.
}

*Corresponding author.

E-mail address: Kristie.Bloom@wits.ac.za

(K. Bloom)

\section{Highlights}

- Chronic infection with HBV is attributed to cccDNA persistence.

- TALENs and miRNA activators have shown anti-HBV therapeutic promise.

- HDR of HBV targets with TALENs and pri-miRNA donors augmented antiviral efficacy.

- Combining gene editing and silencing improves inhibition of HBV gene expression.

\begin{abstract}
Chronic infection with hepatitis B virus (HBV) remains an important global health problem. Currently licensed therapies have modest curative efficacy, which is as a result of their transient effects and limited action on the viral replication intermediate comprising covalently closed circular DNA (cccDNA). Gene editing with artificial HBV-specific endonucleases and use of artificial activators of the RNA interference pathway have shown anti-HBV therapeutic promise. Although results from these gene therapies are encouraging, maximizing durable antiviral effects is important. To address this goal, a strategy that entails combining gene editing with homology-directed DNA recombination (HDR), to introduce HBV-silencing artificial primary microRNAs (pri-miRs) into HBV DNA targets, is reported here. Previously described transcription activator-like effector nucleases (TALENs) that target the core and surface sequences of HBV were used to introduce double stranded breaks in the viral DNA. Simultaneous administration of donor sequences encoding artificial promoterless anti-HBV pri-miRs, with flanking arms that were homologous to sequences adjoining the TALENs' targets, augmented antiviral efficacy. Analysis showed targeted integration and the length of the flanking homologous arms of donor DNA had a minimal effect on antiviral efficiency. These results support the notion that gene editing and silencing may be combined to effect improved inhibition of HBV gene expression.
\end{abstract}

Keywords: HBV; TALEN; Artificial primary miRNA; cccDNA 


\section{Introduction}

Hepatitis B virus (HBV) infection is hyper-endemic to Sub-Saharan Africa and East Asia, and remains a global health priority [1]. It is estimated that 240 million people are chronic carriers of the virus and these individuals are at high risk for complicating cirrhosis and hepatocellular carcinoma. Currently licensed therapies for HBV infection include nucleoside analogs, nucleotide analogs and derivatives of interferon alpha (IFN- $\alpha$ ) [2] and [3]. Although these therapies diminish replication of HBV, treatment interruption usually results in relapse with proliferation of the virus. The main reason for the modest cure rate of licensed regimens is that efficacy of the drugs is short-lived and the transcriptional template of HBV, comprising covalently closed circular DNA (cccDNA), is unaffected by available drugs [4] and [5]. Licensed vaccination against the virus usually prevents HBV infection, but has little or no therapeutic benefit. Developing improved methods of countering HBV thus remains an important priority and inactivating cccDNA is essential to cure the infection.

Employing strategies of gene therapy to inactivate HBV replication permanently has recently shown promise (reviewed in Ref. [6]). Gene editing and gene silencing inactivate HBV and both approaches have been shown to be effective without causing toxicity and other unintended off-target effects. Transcription activator-like effector nucleases (TALENs) [7] and [8] and clustered regularly interspaced short palindromic repeats (CRISPR) with CRISPR-associated (Cas) nucleases [9], [10], [11] and [12] have been engineered to target HBV and are capable of introducing mutations at specific sites of viral DNA. Repeated digestion of target viral DNA to form double strand breaks (DSBs) eventually results in introduction of site-specific mutations. Error-prone non-homologous end joining (NHEJ), required to repair cleaved DNA, eventually leads to mutation of the cccDNA. In addition to gene editing, activation of RNA interference (RNAi) may be used to suppress HBV (reviewed in Ref. [13]). Both synthetic and expressed RNAi activators have good efficacy against HBV. Also, expressed HBV-targeting RNAi activators that generate multiple guides may improve antiviral efficacy and prevent the emergence of escape mutants [14].

In dividing cells, such as are often found in the livers of patients chronically infected with $\mathrm{HBV}$, homology directed recombination (HDR) occurs efficiently (reviewed in Refs. [15] and [16]). HDR is based on producing DSBs at specific target sites and simultaneous introduction of 'repairing' donor DNA. The donor DNA typically has flanking arms with sequences that are complementary to those surrounding the DSBs. To augment efficacy of gene therapy against HBV, we investigated the utility of combining gene editing with use of HDR. The strategy was employed to introduce tricistronic artificial anti-HBV primary microRNAs (pri-miRs) [14] into viral target DNA. We used previously described TALENs that target core and surface sequences of the HBV genome [7]. To integrate $X$ targeting pri-miRs into viral DNA, linear donor sequences with flanking homologous regions were also introduced into liver-derived cells. Augmented efficacy and intended target site integration were observed. 


\section{Materials and methods}

\subsection{Plasmids and donor sequences}

HBV-targeting TALENs [7] and artificial pri-miRs [14] have previously been described. Plasmids that included left and right homologous arms (HAs), each comprising $300 \mathrm{bp}$ of HBV sequences upstream and downstream of the C-TALEN and S-TALEN target sites, were generated using standard conditions of PCR and plasmid cloning. The Kapa HiFi Mix (Kapa Biosystems, MA, USA) was used to generate DNA and primer sets for amplification are described in Supplementary Table 1. Briefly, a bipartite PCR approach was used to amplify the $300 \mathrm{bp}$ left HA and right HA of the predicted TALEN cleavage sites. Primers were designed to include unique restriction sites, complementary sequences and disrupting stop codons that were in frame with the viral ORFs. Amplicons were purified and a second primer-free annealing PCR was carried out to join the left and right HAs. The 5 ' ends of the left $\mathrm{HA}$ reverse primers and right $\mathrm{HA}$ forward primers had unique $20 \mathrm{bp}$ complementary sequences to facilitate annealing and extension of the HAs. Complete surface and core donor templates were inserted into pTZ57R/T using the InsTAclone PCR cloning kit (Thermo Scientific, MA, USA) to generate pTZ-C300 and pTZ-S300. The trimeric pri-miR-31/5/8/9 sequence was excised from $\mathrm{pCl}$-pri-miR-31/5/8/9 [14] using $\mathrm{Xbal}$ and $\mathrm{Nhel}$ and inserted into pTZ-C300 and pTZ-S300 at the unique Nhel site to generate pTZ-C300-pri-miR-31/5/8/9 and pTZ-S300-pri-miR-31/5/8/9. Plasmids with and without the pri-miR-31/5/8/9 element were used as templates to amplify linear donors with left and right HAs, each of $50 \mathrm{bp}, 100 \mathrm{bp}$, $150 \mathrm{bp}$ and $300 \mathrm{bp}$. Prior to transfection, donor template strands were purified with the Gene JET PCR Purification Kit (Thermo Scientific, MA, USA).

\subsection{Transfection of liver-derived cells and HBV knockdown analysis by ELISA}

Liver-derived Huh7 and HepG2.2.15 [17] cell lines were used to assess antiviral efficacy and TALEN-mediated pri-miR integration in culture. Linear HDR donor templates, together with plasmids expressing TALENs and the HBV replication-competent plasmid, $\mathrm{pCH}-9 / 3091$ [18] and [19], were used to co-transfect Huh7 cells transiently. Cells were cultured in DMEM (Lonza, Basel, Switzerland) supplemented with 10\% FCS (Gibco ${ }^{\mathrm{TM}}$, Thermo Scientific, MA, USA), penicillin $(100,000 \mathrm{U} / \mathrm{ml})$, streptomycin $(100,000 \mu \mathrm{g} / \mathrm{ml})$, and maintained in a humidified incubator at $37{ }^{\circ} \mathrm{C}$ and $5 \% \mathrm{CO}_{2}$.

One day prior to transfection, Huh7 cells were seeded in 12-well plates at a density of 120,000 cells per well. Cells were transfected using polyethylenimine with the following combinations of plasmids: $200 \mathrm{ng}$ pCH-9/3091, $100 \mathrm{ng}$ pCMV-GFP, $100 \mathrm{ng}$ linear HDR donor sequences of varying lengths, $800 \mathrm{ng}$ of each plasmid expressing the left and right subunits of the TALENs, or $1600 \mathrm{ng}$ of pUC118. Fluorescence microscopy was used to detect GFP expression and confirm equivalent transfection efficiencies. HBsAg concentrations were measured using the Monolisa ${ }^{\mathrm{TM}}$ HBs Ag ULTRA kit (Bio-Rad, CA, USA).

One day prior to transfection, HepG2.2.15 cells were seeded in 6-well plates at a density of 140,000 cells per well. Two hours before transfection, culture medium was collected to measure baseline HBsAg concentrations. Cells were transfected using polyethylenimine with the following combinations of plasmids: 150 ng pCMV-GFP, 250 ng HDR donor templates 
and $2.3 \mu \mathrm{g}$ of each plasmid expressing the left and right subunits of the TALENs, or $4.6 \mu \mathrm{g}$ of pUC118. Cells were maintained under mildly hypothermic conditions $\left(30^{\circ} \mathrm{C}\right.$ and $\left.5 \% \mathrm{CO}_{2}\right)$ [4]. Growth medium was replaced and HBsAg concentrations were measured on days 3, 5 and 7. On day 7 , cells were harvested and a 1:5 dilution reseeded before repeat transfection.

\subsection{Evaluation of targeted integration}

A PCR-based assay was used to confirm that targeted integration occurred at the intended site of the viral DNA. Primers complementary to sequences within the HBV core or surface sequences, but outside of the homology arms of the donor DNA were used to amplify mutated and wild type viral sequences ( Supplementary Table 1). Corroboration of the intended HDR was carried out by sequencing the amplicons (Inqaba Biotechnologies, Pretoria, South Africa). To measure targeted indel formation, HBV DNA was extracted from HepG2.2.15 cells and subjected to analysis using a T7E1 assay as has been described [4].

\subsection{Statistical analysis}

Data are represented as the mean \pm standard error of the mean. Statistical difference was considered significant when $\mathrm{P}<0.05$ and was obtained using the nonparametric Student's $t$ test with the GraphPad Software (GraphPad Software, Inc., CA, USA).

\section{Results}

\subsection{Using TALENs to introduce artificial anti-HBV pri-miRs into viral DNA}

Dimeric engineered nucleases were generated to cleave sequences within the core and surface open reading frames of HBV [7], and are termed C-TALEN and S-TALEN respectively (Fig. 1A). The left and right subunits that constitute the TALENs bind two sequences of 19 nucleotides, each with a T residue at the $5^{\prime}$ end. Fokl nuclease domains were positioned at the C-terminal end of each of the TALEs and the dimers designed to be oriented in a headto-head arrangement with an intervening spacer of $13 \mathrm{bp}$. The complete TALEN subunits each also included a nuclear localization signal (NLS). Trimeric pri-miRs generate silencing sequences with cognates in the $X$ ORF ( Fig. $1 \mathrm{~A}$ ) and the artificial gene silencers have a scaffold derived from pri-miR-31 [14]. 
A

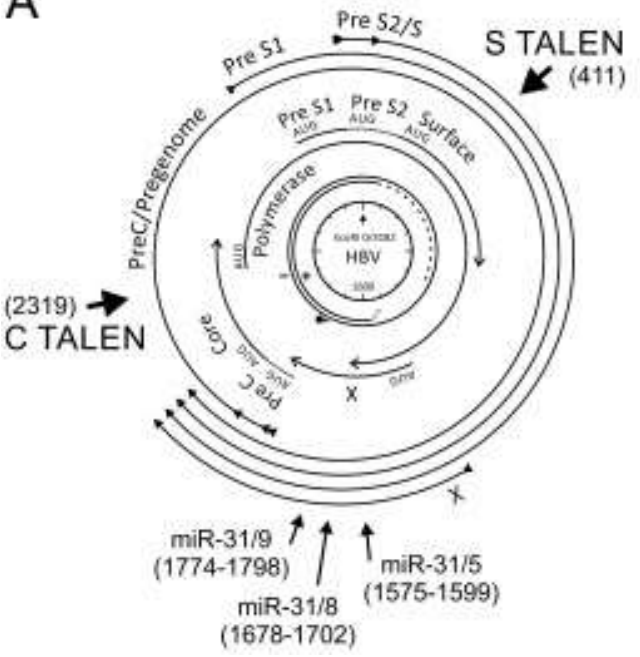

B

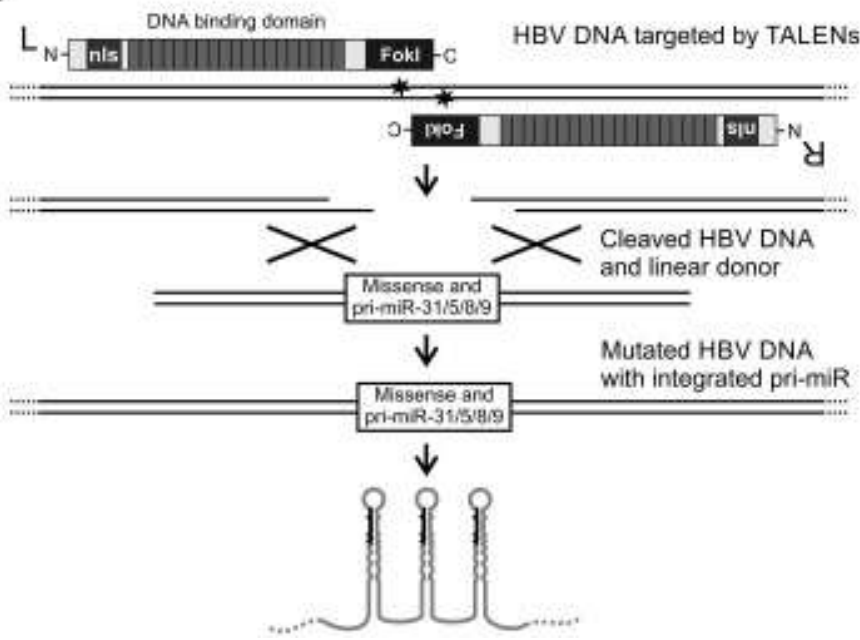

Fig. 1. HBV-targeting TALENs, pri-miRs and rationale of the mechanism of antiviral homology-directed recombination (HDR). A. The HBV genome, comprising relaxed circular DNA (rCDNA) with ' + ' and ' - ' strands is indicated at the center. Coordinates of the nucleotides are calculated relative to the unique EcoRI restriction site. The four open reading frames (ORFs) of the virus, core, polymerase, surface and $X$, are depicted as surrounding arrows. The four main HBV transcripts, with common $3^{\prime}$ ends, are shown as concentric outermost arrows. Sites of action by the C-TALEN and S-TALEN are shown with coordinates of nucleotides that are central to the spacers between the two dimers given in parentheses. Artificial pri-miRs targeting the $X$ ORF are also shown with viral sequences complementary to the mature guides given in parentheses. B. Schematic illustration of dimeric TALEN-mediated induction of double stranded breaks at the viral DNA. Each subunit of the TALENs includes a nuclear localization signal (nls), DNA-binding domain and endonuclease (Fokl) domain. Subsequent HDR involving the linear donor template incorporates the promoterless pri-miR-31/5/8/9 trimeric cassette with missense sequences.

The principle underlying introduction of pri-miR-31/5/8/9 sequences into HBV DNA is based on improved efficiency of HDR in dividing cells following formation of DSBs in the target DNA (reviewed in Refs. [15] and [16]). In our scheme, the process is initiated by cleavage of HBV DNA by the TALENs (Fig. 1B). Introduction of linear donor DNA with homologous viral sequences flanking the cleavage site enables HDR. As the donor also contains a sequence encoding HBV-targeting pri-miR-31/5/8/9, an additional antiviral effector is introduced to augment efficacy. The inserts are promoterless and transcription of the artificial pri-miRs is driven by Pol II action from the viral promoters. Processing of the trimeric pri-miR-31/5/8/9 should then proceed according to canonical pathways of miR maturation. In addition, missense sequences in the inserts further disrupt expression of viral genes following integration.

\subsection{Donors for HDR with varying flanking homology arms}

Evidence indicates that linear donor duplexes are better recognized and incorporated at a DNA break than are plasmid templates [21]. Moreover, the length of a donor template strand's homologous regions affect HDR efficiency. To assess the influence of the flanking arms' length, a range of linear sequences, generated using PCR, was used (Fig. 2). The sequences included $50 \mathrm{bp}, 100 \mathrm{bp}, 150 \mathrm{bp}$ or $300 \mathrm{bp}$ at each of the upstream and downstream ends, which were homologous to the HBV sequences surrounding the TALEN cleavage sites. Amplicons included disruptive missense elements (Fig. 2A and B) or a combination of the missense and artificial pri-miR-31/5/8/9-encoding DNA (Fig. 2C and D). 
Sizes of the amplicons were determined by use of amplifying primers that were complementary to appropriately positioned sites on the PCR template. Amplifications occurred efficiently, and appropriately sized linear fragments were generated for the donors containing missense (Fig. 2B) or missense with pri-miR-31/5/8/9-encoding elements (Fig. 2D).
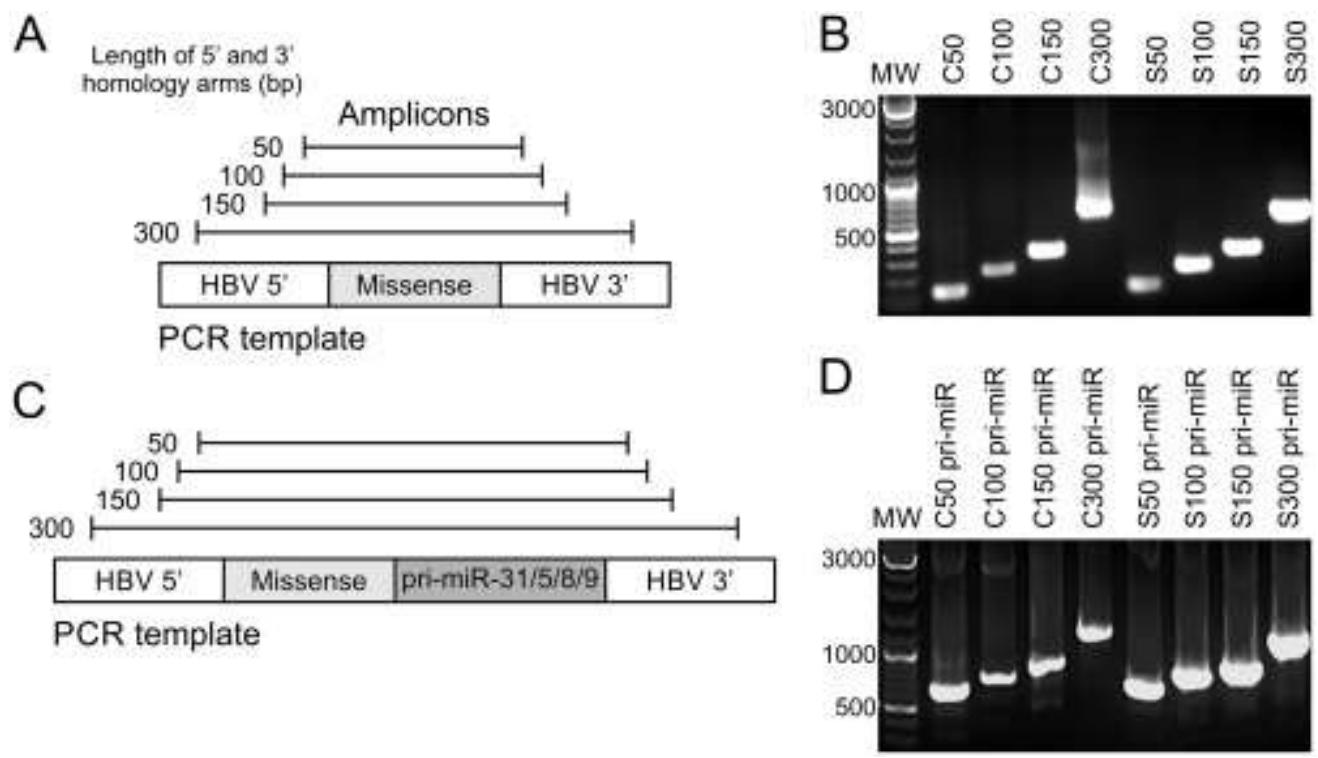

Fig. 2. PCR-based generation of donor sequences containing missense sequences with or without pri-miR31/5/8/9 trimeric cassettes. A. Schematic of PCR template used to amplify donor sequences of varying lengths. The indicated amplicons have flanking arms that are defined by the positions of the sequences that are complementary to the amplifying oligonucleotides. The length of the upstream and downstream homologous sequences are indicated. B. Agarose gel electrophoresis to verify yield and size of amplicons with arms flanking the site of action of the C-TALEN (C50, C100, C150 and C300) and S-TALEN (S50, S100, S150 \& S300). C \& D. Scheme to indicate PCR amplification and yields as described in A \& B but with the additional cassette encoding the pri-miR-31/5/8/9 cassette.

\subsection{Evaluation of effects of donor sequences without TALENs}

As an initial assessment, markers of viral replication were measured following transfection of Huh7 cells (Fig. 3A) or HepG2.2.15 cells (Fig. 3B) with each of the panel of donor sequences without TALENs. In the case of analysis in Huh7 cells, a replication-competent plasmid was included to drive HBV proliferation. As expected, transfection with plasmids encoding inactive TALENs or those targeting core, had no effect on secretion of HBV surface antigen (HBsAg). In contrast the plasmid expressing the antiviral pri-miR-31/5/8/9 from the CMV promoter and surface-targeting TALEN each caused significant inhibition of the marker of HBV replication in both cell lines. Linear donor DNA strands do not contain promoters, and any decrease in HBsAg expression may be the result of a non-specific effect or unintended integration into transcriptionally active sequences. In Huh7 cells, sequences containing the trimeric pri-miR element caused modest inhibition of HBsAg secretion ( Fig. 3A), which was less apparent after transfection of HepG2.2.15 cells (Fig. 3B). These observations suggest that integration of the donors into HBV-replicating $\mathrm{pCH}-9 / 3091$ plasmid, absent from the HepG2.2.15 cells, may account for the inhibition of $\mathrm{HBsAg}$ secretion [17] and [18]. In both cell lines, and with each of the panel of donor sequences that did not contain the artificial pri-miR sequence, inhibition of HBV replication was absent. 


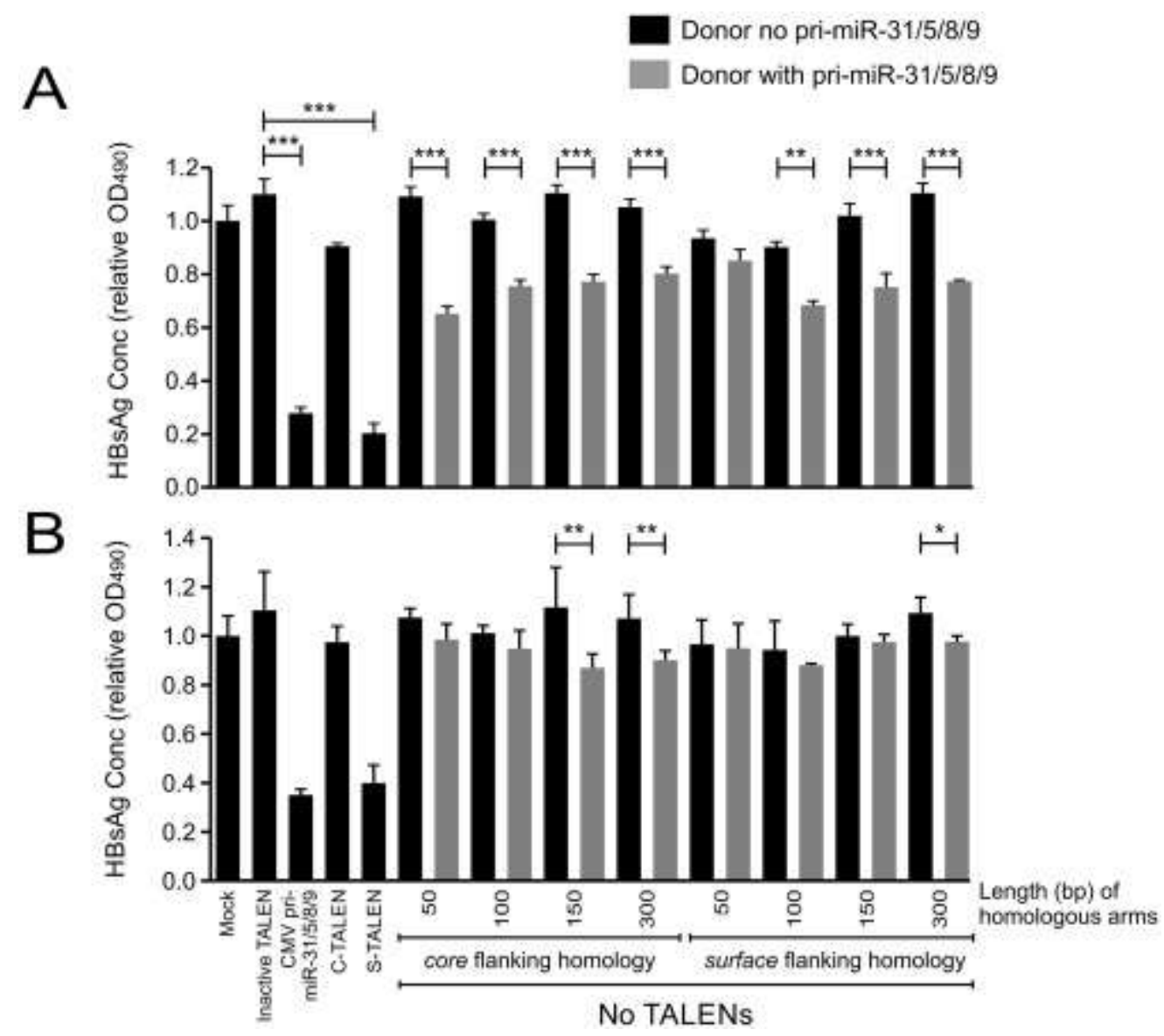

Fig. 3. Effect of donor sequences on $\mathrm{HBV}$ surface antigen (HBsAg) secretion from transfected liver derived cells. A. Huh7 cells and B. HepG2.2.15 cells transfected with a panel of core and surface donor sequences with varying homology arm lengths in the absence of TALENs. Control groups included cells that had been mock treated, transfected with plasmids containing an active CMV-driven pri-miR-31/5/8/9, an inactive TALEN, CTALEN or S-TALEN. Error bars represent the standard error of the mean $(n=6)$. Analysis of data was carried out using the standard student t-test and statistical significance is indicated $(*: p<0.05, * *: p<0.01$ and $* * *: p<0.001)$.

\subsection{Augmented antiviral efficacy when using TALENs in combination with donors for HDR}

Initial evaluation of effects of combinations of donors and TALENs were carried out on Huh7 cells. Transfection of the C-TALEN-encoding plasmid alone or in combination with donor sequences lacking the pri-miR-31/5/8/9 sequence did not cause any decrease in the concentration of HBsAg in the culture supernatant (Fig. 4A). However, when the donor sequences containing the pri-miR-31/5/8/9 element were co-transfected, significant decreases in concentrations of HBsAg in the culture supernatants were observed. Analysis following transfections with plasmids expressing the S-TALEN confirmed that HBsAg concentration in the culture supernatant was decreased. This was observed regardless of whether the donor contained the pri-miR-31/5/8/9 sequence or not. Donor sequences with shorter flanking arms had moderately improved antiviral efficacy in transfected Huh7 cells. For subsequent evaluation in HepG2.2.15 cells, the donors with arms comprising $100 \mathrm{bp}$ of upstream and downstream homology were used. 

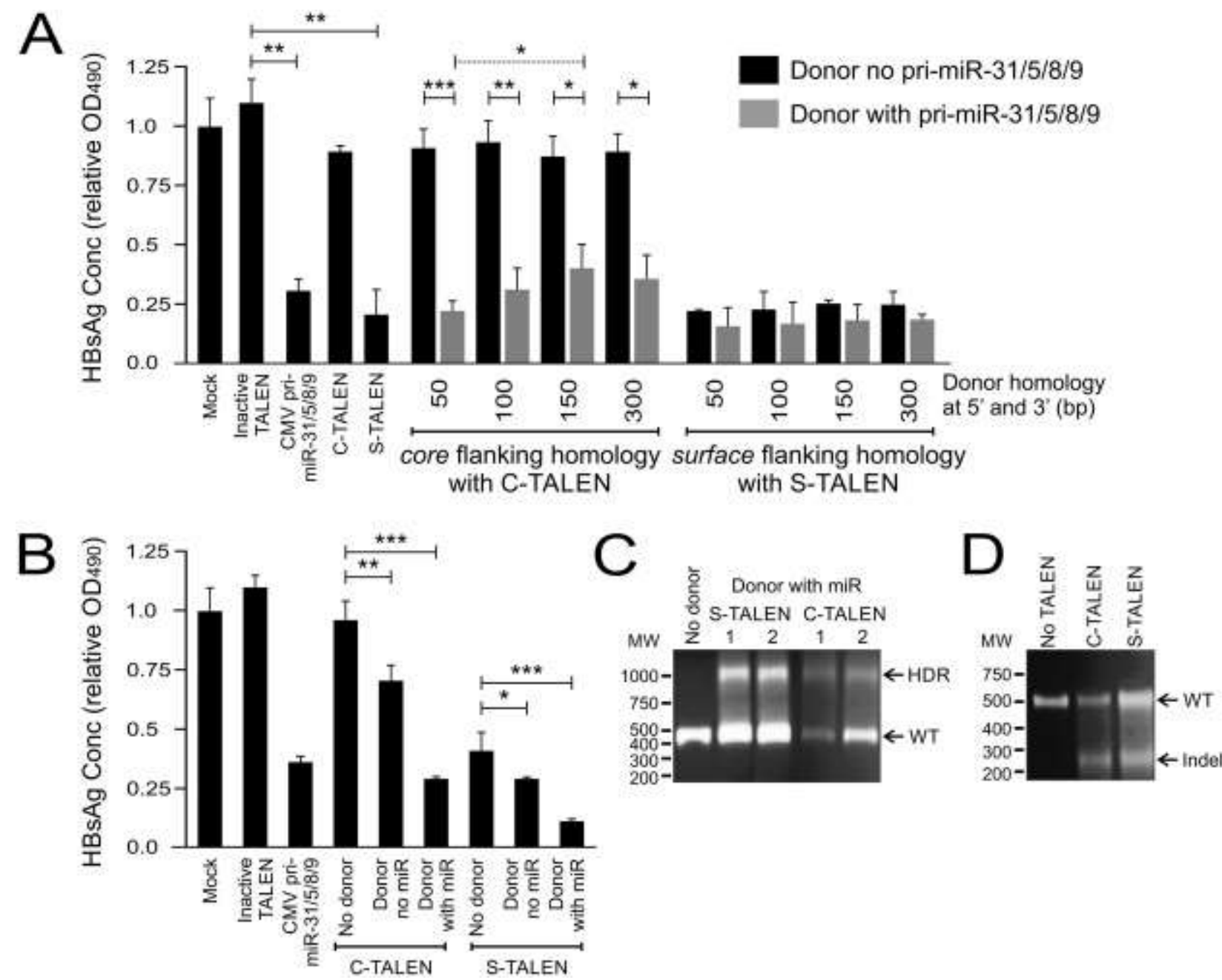

Fig. 4. Effects of combinations of TALENs and donors on inhibition of HBV surface antigen (HBsAg) and targeted mutation. A. Secretion of HBsAg from Huh7 cells that had been co-transfected with combinations of C-TALEN or S-TALEN together with donors of varying sizes. Controls were similar to those described in the legend to Fig. 3. B. Concentrations of HBsAg in culture supernatants of HepG2.2.15 cells that had been transfected with the indicated TALENs, and donor sequences with or without the pri-miR-31/5/8/9 sequence. Flanking arms of the donors comprised two 100 bp sequences that were complementary to HBV DNA upstream and downstream of the cleavage site. C. Duplicate PCR-based analysis to verify targeted insertion following transfection with S-TALEN or C-TALEN and donor containing the pri-miR-31/5/8/9 sequence. As above, flanking arms of the donors comprised two 100 bp sequences that were complementary to HBV $5^{\prime}$ and $3^{\prime}$ of the cleavage site. D. T7E1 assay to verify targeted indel formation following action of C-TALEN and STALEN.

Analysis following transfection of HepG2.2.15 cells corroborated the interpretation that HDR augmented anti-HBV efficacy of TALENs (Fig. 4B). Replication of HBV in these cells is driven from the greater-than-genome length replication-competent viral integrants and also the cccDNA that is produced during viral replication. Transfection of the plasmid expressing the C-TALEN alone did not have an effect, and inclusion of a donor without the RNAi activator caused a modest but significant decrease in HBsAg concentration in the cell culture supernatant. Transfection of a donor that had the artificial RNAi activator caused a more significant decrease of $\mathrm{HBsAg}$, and the concentration of the viral antigen was approximately $30 \%$ of the control. As expected, transfecting plasmid expressing the S-TALEN alone inhibited HBsAg secretion. The donor without pri-miR-31/5/8/9 sequence caused a further 
decrease in the viral replication marker and the inhibitory effect was augmented following co-transfection of the donor containing the anti-HBV trimeric RNAi activator. Collectively these results are consistent with the interpretation that the S-TALEN alone is capable of introducing mutations at the viral surface sequence, which in turn leads to diminished secretion of the viral antigen. Including donors to activate HDR and disrupt the viral targets augmented the inhibitory effects observed on the viral markers of replication. As expected, the C-TALEN alone does not disrupt the surface sequence and consequently HBsAg secretion into the culture supernatant is unaffected. However, combination of the C-TALEN with a donor sequence containing the pri-miR-31/5/8/9 element decreased HBsAg secretion, which is as a result of the artificial RNAi activator targeting the viral mRNA.

To verify that HDR had occurred in transfected HepG2.2.15 cells according to the intended mechanism, a PCR assay was employed to identify integration events. Primers that flanked the intended site of integration were used, which would amplify wild type and mutated sequences. Amplification of DNA extracted from cells transfected with TALEN-expressing plasmids with or without pri-miR-31/5/8/9 sequence revealed bands with sizes of approximately $500 \mathrm{bp}$ and $1 \mathrm{~kb}$ (Fig. 4C). The band of $500 \mathrm{bp}$ is the expected size of the HBV sequence without HDR, while the band of $1 \mathrm{~kb}$ is anticipated following HDR with incorporation of the sequence containing the pri-miR-31/5/8/9 element. Sequencing the amplicons confirmed that integration of the donor sequences had occurred at the TALENs' cleavage sites (Supplementary Fig. 1). Verification that the C-TALEN and S-TALEN caused targeted indels in the absence of donors was confirmed following analysis using a T7E1 assay (Fig. 4D). Taken together these results indicate that DSBs introduced by HBV-targeting TALENs induce error-prone NHEJ in the absence of donor DNA, while HDR is facilitated by the engineered nucleases when donor sequences are introduced into the cells.

\section{Discussion}

Chronic infection with HBV remains an important global health problem [1]. Available therapies have modest curative efficacy and there is a need for development of new approaches to eliminating HBV from chronic carriers of the virus [2] and [3]. Central to the limitations of current treatments is the inability to eliminate the cccDNA from infected hepatocytes. Gene editing has recently shown promise as the means of inactivating cccDNA to achieve a cure from the infection (reviewed in Ref. [6]). The basis of using engineered nucleases to disable cccDNA is that repeated digestion of the target viral DNA eventually results in mutation following error-prone repair of the viral sequences through activation of the NHEJ pathway.

HDR is an alternative mutagenic mechanism that relies on target cleavage and recombination involving donor sequences with arms of homology that flank the cleavage site (reviewed in Refs. [15] and [16]). The mechanism is efficient in dividing cells and has been widely exploited for insertion of sequences at particular target sites. In patients with chronic HBV infection, rapid turnover of hepatocytes is a feature that is particularly prominent in cases where complicating cirrhosis occurs. In this study, we utilized HDR to improve antiviral efficacy and mutagenesis of HBV sequences. The procedure entailed using 
a combination of TALENs that cleave core and surface sequences of HBV, with linear donor DNA that includes missense sequences and antiviral trimeric artificial pri-miRs. Introducing these elements into cultured cells that replicate HBV resulted in more effective inhibition of replication of the virus. In cells where HDR had occurred, inhibition of viral replication should thus result from a combination of post transcriptional gene silencing as well as mutation of viral DNA.

Although use of gene editing to disable HBV permanently is promising, some challenges remain. Especially important are minimizing unintended off target effects, delivering the effectors to HBV-infected cells efficiently and attenuating any immune response to the gene editors. These topics are also a priority of other areas of gene therapy and progress is steadily being made to overcome the hurdles. Viral and non-viral vectors are both being developed to achieve the goal of efficient delivery of therapeutic sequences, and the field is currently at an interesting stage of development. A remarkable feature of naturally occurring pri-miRs is that they are often secreted from cells in stable exosomes and some evidence indicates that the exosome-containing miRs are mediators of intercellular signaling [19] and [20]. It is tempting to surmise that anti-HBV miRs, generated from viral DNA engineered using HDR, may be processed similarly to effect a paracrine antiviral effect.

\section{Acknowledgements}

This work was supported by the South African Medical Research Council, Poliomyelitis Research Foundation,Johnson \& Johnson Innovation, Claude Leon Foundation and South African National Research Foundation (81768, 81692, 68339, 85981 \& 77954).

\section{Appendix A. Supplementary data}

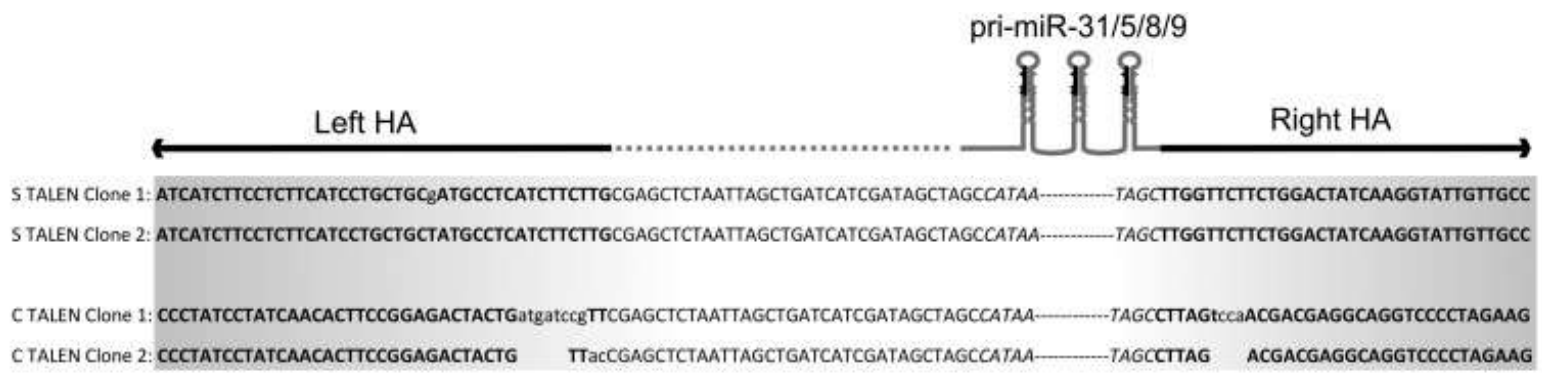

Supplementary Figure 1. Targeted integration of the pri-miR-31/5/8/9 sequence in HepG2.2.15 cells with S- and C-TALEN. Key features are represented graphically abswe the S- und C TALEN sequences Regions complementary to the left and right homology ams (HA) are shown in bold, and include the respective TALEN binding sites. The 5' and 3' regions of the integated pri-miR-31/5/8:9 sequences are indicated in italics. 


\section{References}

[1] WHO, Hepatitis B Virus Fact Sheet No. 204 (Updated July 2013), 2013.

[2] S. Chevaliez, C. Hezode, S. Bahrami, M. Grare, J.M. Pawlotsky, Long-term hepatitis B surface antigen ( $\mathrm{HBsAg}$ ) kinetics during nucleoside/nucleotide analogue therapy: finite treatment duration unlikely, J. Hepatol. 58 (2013) $676 \mathrm{e} 683$.

[3] C. Trepo, H.L. Chan, A. Lok, Hepatitis B virus infection, Lancet 384 (2014) $2053 e 2063$.

[4] M. Nassal, HBV cccDNA: viral persistence reservoir and key obstacle for a cure of chronic hepatitis B, Gut 64 (2015) 1972e1984.

[5] D. Halegoua-De Marzio, H.-W. Hann, Then and now: the progress in hepatitis B treatment over the past 20 years, World J. Gastroenterol. 20 (2014) 401e413.

[6] A. Ely, B. Moyo, P. Arbuthnot, Progress with developing use of gene editing to cure chronic infection with hepatitis B virus, Mol. Ther. 24 (2016) 671e677.

[7] K. Bloom, A. Ely, C. Mussolino, T. Cathomen, P. Arbuthnot, Inactivation of hepatitis B virus replication in cultured cells and in vivo with engineered transcription activator-like effector nucleases, Mol. Ther. 21 (2013) 1889e1897.

[8] J. Chen, W. Zhang, J. Lin, F. Wang, M. Wu, C. Chen, Y. Zheng, X. Peng, J. Li, Z. Yuan, An efficient antiviral strategy for targeting hepatitis $B$ virus genome using Transcription Activator-Like Effector Nucleases, Mol. Ther. 22 (2013) 303e311.

[9] S.R. Lin, H.C. Yang, Y.T. Kuo, C.J. Liu, T.Y. Yang, K.C. Sung, Y.Y. Lin, H.Y. Wang, C.C. Wang, Y.C. Shen, F.Y. Wu, J.H. Kao, D.S. Chen, P.J. Chen, The CRISPR/cas9 system facilitates clearance of the intrahepatic HBV templates in vivo, Mol. Ther. Nucleic Acids 3 (2014) e186.

[10] S. Zhen, L. Hua, Y.H. Liu, L.C. Gao, J. Fu, D.Y. Wan, L.H. Dong, H.F. Song, X. Gao, Harnessing the clustered regularly interspaced short palindromic repeat (CRISPR)/CRISPRassociated Cas9 system to disrupt the hepatitis B virus, Gene Ther. 22 (2015) 404e412.

[11] J. Wang, Z.W. Xu, S. Liu, R.Y. Zhang, S.L. Ding, X.M. Xie, L. Long, X.M. Chen, H. Zhuang, F.M. Lu, Dual gRNAs guided CRISPR/Cas9 system inhibits hepatitis B virus replication, World J. Gastroenterol. 21 (2015) 9554e9565.

[12] C. Seeger, J.A. Sohn, Targeting hepatitis B virus with CRISPR/cas9, Mol. Ther. Nucleic Acids 3 (2014) e216.

[13] D. Ivacik, A. Ely, P. Arbuthnot, Countering hepatitis B virus infection using RNAi: how far are we from the clinic? Rev. Med. Virol. 21 (2011) 383e396.

[14] A. Ely, T. Naidoo, P. Arbuthnot, Efficient silencing of gene expression with modular trimeric Pol II expression cassettes comprising microRNA shuttles, Nucleic Acids Res. 37 (2009) e91. 
[15] T. Gaj, C.A. Gersbach, C.F. Barbas III, ZFN, TALEN, and CRISPR/Cas-based methods for genome engineering, Trends Biotechnol. 31 (2013) 397e405.

[16] C. Wei, J. Liu, Z. Yu, B. Zhang, G. Gao, R. Jiao, TALEN or Cas9-rapid, efficient and specific choices for genome modifications, J. Genet. Genomics 40 (2013) 281e289.

[17] K. Yoon, A. Cole-Strauss, E.B. Kmiec, Targeted gene correction of episomal DNA in mammalian cells mediated by a chimeric RNA.DNA oligonucleotide, Proc. Natl. Acad. Sci. U. S. A. 93 (1996) 2071e2076.

[18] P.P. Chan, M. Lin, A.F. Faruqi, J. Powell, M.M. Seidman, P.M. Glazer, Targeted correction of an episomal gene in mammalian cells by a short DNA fragment tethered to a triplexforming oligonucleotide, J. Biol. Chem. 274 (1999) 11541e11548.

[19] C.H. Lawrie, S. Gal, H.M. Dunlop, B. Pushkaran, A.P. Liggins, K. Pulford, A.H. Banham, F. Pezzella, J. Boultwood, J.S. Wainscoat, C.S. Hatton, A.L. Harris, Detection of elevated levels of tumour-associated microRNAs in serum of patients with diffuse large B-cell lymphoma, Br. J. Haematol. 141 (2008) 672e675.

[20] J. Skog, T. Wurdinger, S. van Rijn, D.H. Meijer, L. Gainche, M. Sena-Esteves, W.T. Curry Jr., B.S. Carter, A.M. Krichevsky, X.O. Breakefield, Glioblastoma microvesicles transport RNA and proteins that promote tumour growth and provide diagnostic biomarkers, Nat. Cell Biol. 10 (2008) 1470e1476.

[21] S.J. Orlando, Y. Santiago, R.C. DeKelver, Y. Freyvert, E.A. Boydston, E.A. Moehle, V.M. Choi, S.M. Gopalan, J.F. Lou, J. Li, J.C. Miller, M.C. Holmes, P.D. Gregory, F.D. Urnov, G.J. Cost, Zinc-finger nuclease-driven targeted integration into mammalian genomes using donors with limited chromosomal homology, Nucl. Acids Res. 38 (2010) e152. 